\title{
Front Matter: Volume 9516
}

, "Front Matter: Volume 9516," Proc. SPIE 9516, Integrated Optics: Physics and Simulations II, 951601 (19 May 2015); doi: 10.1117/12.2197515

SPIE. Event: SPIE Optics + Optoelectronics, 2015, Prague, Czech Republic 


\title{
PROCEEDINGS OF SPIE
}

\section{Integrated Optics: Physics and Simulations II}

\author{
Pavel Cheben \\ Jiří Čtyroký \\ Iñigo Molina-Fernández \\ Editors
}

13-15 April 2015

Prague, Czech Republic

Sponsored by

SPIE

Cooperating Organisations

HiPER Project (United Kingdom)

ELI Beamlines (Czech Republic)

Laserlab Europe

Published by

SPIE

Volume 9516 
The papers included in this volume were part of the technical conference cited on the cover and title page. Papers were selected and subject to review by the editors and conference program committee. Some conference presentations may not be available for publication. The papers published in these proceedings reflect the work and thoughts of the authors and are published herein as submitted. The publisher is not responsible for the validity of the information or for any outcomes resulting from reliance thereon.

Please use the following format to cite material from this book:

Author(s), "Title of Paper," in Integrated Optics: Physics and Simulations II, edited by Pavel Cheben, Jiř́ Čtyroký, Iñigo Molina-Fernández, Proceedings of SPIE Vol. 9516 (SPIE, Bellingham, WA, 2015) Article CID Number.

ISSN: 0277-786X

ISBN: 9781628416374

Published by

SPIE

P.O. Box 10, Bellingham, Washington 98227-0010 USA

Telephone +1 3606763290 (Pacific Time) · Fax +1 3606471445

SPIE.org

Copyright (C) 2015, Society of Photo-Optical Instrumentation Engineers.

Copying of material in this book for internal or personal use, or for the internal or personal use of specific clients, beyond the fair use provisions granted by the U.S. Copyright Law is authorized by SPIE subject to payment of copying fees. The Transactional Reporting Service base fee for this volume is $\$ 18.00$ per article (or portion thereof), which should be paid directly to the Copyright Clearance Center (CCC), 222 Rosewood Drive, Danvers, MA 01923. Payment may also be made electronically through CCC Online at copyright.com. Other copying for republication, resale, advertising or promotion, or any form of systematic or multiple reproduction of any material in this book is prohibited except with permission in writing from the publisher. The CCC fee code is 0277-786X/15/\$18.00.

Printed in the United States of America.

Publication of record for individual papers is online in the SPIE Digital Library.

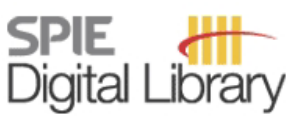

SPIEDigitalLibrary.org

Paper Numbering: Proceedings of SPIE follow an e-First publication model, with papers published first online and then in print. Papers are published as they are submitted and meet publication criteria. A unique citation identifier (CID) number is assigned to each article at the time of the first publication. Utilization of CIDs allows articles to be fully citable as soon as they are published online, and connects the same identifier to all online, print, and electronic versions of the publication. SPIE uses a six-digit CID article numbering system in which:

- The first four digits correspond to the SPIE volume number.

- The last two digits indicate publication order within the volume using a Base 36 numbering

system employing both numerals and letters. These two-number sets start with 00, 01, 02, 03,

$04,05,06,07,08,09,0 \mathrm{~A}, 0 \mathrm{~B} \ldots \mathrm{OZ}$, followed by 10-1Z, 20-2Z, etc.

The CID Number appears on each page of the manuscript. The complete citation is used on the first page, and an abbreviated version on subsequent pages. 


\title{
Contents
}

\author{
vii Authors \\ ix Conference Committee
}

\section{SESSION 1 PHOTONIC INTEGRATION}

951602 Towards an automated design framework for large-scale photonic integrated circuits (Invited Paper) [9516-1]

951603 Passive and electro-optic polymer photonics and InP electronics integration (Invited Paper) [9516-2]

951604 Rethinking the surface of optical waveguides (Invited Paper) [9516-3]

951605 Simulation of self-organized waveguides for self-aligned coupling between micro- and nano-scale devices [9516-4]

\section{SESSION 2 DEVICES AND CIRCUITS I}

951606 Nonlinear optical signal processing in high figure of merit CMOS compatible platforms (Invited Paper) [9516-5]

951607 High-speed and low-power silicon-organic hybrid modulators for advanced modulation formats (Invited Paper) [9516-6]

951608 A numerical investigation of silicon-based optical sampling [9516-7]

\section{SESSION 3 DEVICES AND CIRCUITS II}

951609 Silicon nanophotonic integrated devices enabling multiplexed on-chip optical interconnects (Invited Paper) [9516-8]

9516 OB Expanding sampling in a SWIFTS-Lippmann spectrometer using an electro-optic MachZehnder modulator [9516-10]

$95160 \mathrm{C}$ High resolution TE\&TM near infrared compact spectrometer based on waveguide grating structures [9516-11]

9516 OD Photovoltaic maximum power point search method using a light sensor [9516-12] 
$9516 \mathrm{OH} \quad$ Plasmonic nanoantenna coherent absorption switches for integrated photonics (Invited Paper) [9516-16]

\section{SESSION 5 SUBWAVELENGTH STRUCTURES}

9516 Ol High-efficiency fully etched fiber-chip grating couplers with subwavelength structures for datacom and telecom applications [9516-17]

$95160 \mathrm{~J} \quad$ High efficiency blazed fiber-chip grating coupler with interleaved trenches [9516-18]

9516 OK Fiber-chip edge coupler with large mode size for silicon photonic wire waveguides [9516-19]

$9516 \mathrm{OL}$ A subwavelength structured multimode interference coupler for the 3-4 $\mu \mathrm{m}$ mid-infrared band (Best Student Paper Award) [9516-20]

$95160 \mathrm{M}$ Simulations of waveguide Bragg grating filters based on subwavelength grating waveguide [9516-21]

\section{SESSION 6 THEORY, SIMULATION, AND DESIGN}

$95160 \mathrm{~N} \quad$ Circuit modeling based optimization of high speed carrier depletion silicon modulators [9516-22]

$951600 \quad$ Modeling of anisotropic grating structures with active dipole layers [9516-23]

\section{SESSION 7 MATERIALS AND FABRICATION}

$95160 T \quad$ Epitaxially grown vertical junction phase shifters for improved modulation efficiency in silicon depletion-type modulators [9516-29]

9516 OV Photoluminescence of graphene oxide integrated with silicon substrates [9516-31]

9516 OW Pixel isolation in Type-II InAs/GaSb superlattice photodiodes by femto-second laser annealing [9516-32]

\section{POSTER SESSION}

9516 OX Algorithm applying a modified BRDF function in $\Lambda$-ridge concentrator of solar radiation [9516-26]

$95160 Z$ Optimization design of pulse compression multilayer dielectric gratings [9516-36]

951610 Ultra-long photonic nanojet formed by dielectric cubes [9516-37] 
951612 Electro-optic 1x2 switch based on proton-exchanged channel waveguides in $\mathrm{LiNbO}_{3}$ [9516-40]

951614 Estimation of the sinusoidal oscillation parameters in the adaptive optics system based on the example of the photovoltaic system [9516-42]

951615 Terahertz material characterization for nonreciprocal integrated optics [9516-44]

951616 Magnetoplasmonic waveguiding structure with nonreciprocal dispersion of guided TM modes [9516-45]

951617 Light trap with reactive sun tracking for high-efficiency spectrum splitting photovoltaic conversion [9516-46]

951618 Transparency-switching optical element for sun tracking applications [9516-47]

951619 Side band suppression for wide band optical RoF systems [9516-48]

$95161 \mathrm{~A}$ Femtosecond writing of depressed cladding waveguides in strongly cumulative regime [9516-49] 


\title{
Authors
}

Numbers in the index correspond to the last two digits of the six-digit citation identifier (CID) article numbering system used in Proceedings of SPIE. The first four digits reflect the volume number. Base 36 numbering is employed for the last two digits and indicates the order of articles within the volume. Numbers start with 00, 01, 02, 03, 04, 05, 06, 07, 08, 09, OA, OB...0Z, followed by 10-1Z, 20-2Z, etc.

\author{
Alonso-Ramos, Carlos A., OJ, OL \\ Annoni, A., 04 \\ Apostoleris, Harry, 17, 18 \\ Arellano, Cristina, 02 \\ Avramopoulos, H., 03 \\ Bach, H.-G., 03 \\ Bai, Liang, $\mathrm{OZ}$ \\ Ben Abid, Samir, 19 \\ Benech, Pierre, OB, OC \\ Benedikovic, Daniel, OI, OJ, OK \\ Beretta, A., 03 \\ Bolten, J., 07 \\ Bonneville, Christophe, $\mathrm{OB}$ \\ Borriello, A., OV \\ Bruck, Roman, $\mathrm{OH}$ \\ Buca, D., OT \\ Bukharin, Mikhail A., 1A \\ Cangini, G., 03 \\ Casalino, M., OV \\ Chang, Li-Jen, 10 \\ Cheben, Pavel, OI, OJ, OK, OL \\ Chen, Lawrence, OM \\ Chen, Sitao, 09 \\ Chiesa, Matteo, 17, 18 \\ Choi, J. H., 03 \\ Coppola, G., OV \\ Courjal, N., OC \\ Čtyroký, Jiří, OM \\ Dado, Milan, Ol, OJ \\ Dagens, B., 16 \\ Dai, Daoxin, 09 \\ Dalton, L. R., 07 \\ Dardano, P., OV \\ Das, Sona, OW \\ Das, Utpal, OW \\ de Mengin, Mikhaël, OB, OC \\ De Stefano, L., OV \\ Dede, Alberto, 03 \\ Delâge, André, OK \\ Dinu, R., 03 \\ Drouhin, H.-J., 00 \\ Dupuy, J. Y., 03 \\ Elder, D. L., 07 \\ Fan, Shuwei, $\mathrm{OZ}$ \\ Fédéli, J.-M., OJ \\ Fedorov, V. A., 12 \\ Felipe, D., 03 \\ Fördös, Tibor, 00 \\ Freude, W., 07
}

\author{
Gautam, Nutan, OW \\ Giesecke, A. L., 07 \\ Giordano, M., OV \\ Glesk, Ivan, OM \\ Gonthiez, Thierry, OB \\ Grillanda, S., 04 \\ Groumas, P., 03 \\ Halagačka, Lukas, 00, 16 \\ Halir, Robert, OI, OJ, OK, OL \\ Harati, P., 03 \\ Heidmann, Samuel, OB, OC \\ Horák, Tomáš, 15 \\ Hosseini, Seyedreza, ON \\ Hraghi, Abir, 19 \\ Jaffrès, Henri, 00 \\ Jamshidi, Kambiz, 08, 0N \\ Janz, Siegfried, OI, OK \\ Jazayerifar, M., 08 \\ Jia, Shenli, OZ \\ Jorge, F., 03 \\ Kania, Dariusz, 14 \\ Katopodis, V., 03 \\ Keil, N., 03 \\ Kern, P., OC \\ Khudyakov, Dmitriy V., $1 \mathrm{~A}$ \\ Koeber, S., 07 \\ Koenigsmann, M., 07 \\ Kohler, M., 07 \\ Koltchanov, Igor, 02 \\ Konczykowska, A., 03 \\ Koos, C., 07 \\ Korkishko, Yu. N., 12 \\ Kostritskii, S. M., 12 \\ Kouloumentas, Ch., 03 \\ Krishna, Sanjay, OW \\ Kwiecien, Pavel, OM \\ Lampin, Jean-François, 15 \\ Lapointe, Jean, 0l \\ Lavermann, M., 07 \\ Le Coarer, Etienne, OB, OC \\ Leuthold, J., 07 \\ Lilliu, Samuele, 18 \\ Liu, Cheng-Yang, 10 \\ Loridat, Joran, $\mathrm{OB}$ \\ Maese-Novo, A., 03 \\ Malsam, D., 07 \\ Martin, Guillermo, OB, OC \\ Mashanovich, Goran Z., OL \\ Melati, D., 04
}


Melloni, A., 04

Menif, Mourad, 19

Merget, F., OT

Mičica, Martin, 15

Miller, E., 03

Mingaleev, Sergei, 02

Molina-Fernández, Íñigo, OJ, OL

Morand, Alain, OB, OC

Morandotti, R., 06

Morichetti, F., 04

Moscoso-Mártir, A., OT

Moss, D. J., 06

Muskens, Otto L., $\mathrm{OH}$

Nedeljkovic, Milos, $\mathrm{OL}$

Nodjiadjim, V., 03

Ortega-Moñux, Alejandro, OI, OJ, OK, OL

Ostrowski, Mariusz, OD

Palmer, R., 07

Papes, Martin, OK

Pištora, Jaromír, 00, 15, 16

Plachta, Kamil, OX

Postava, Kamil, 00, 15, 16

Ramos, Carlos A., OK

Rea, I., OV

Rendina, I., OV

Richter, André, 02

Richter, Ivan, OM

Romero-García, S., OT

Sánchez-Postigo, Alejandro, OL

Sansone, L., OV

Schell, Martin, 03

Schindler, P. C., 07

Schmid, Jens H., Ol, OJ, OK

Sharif Azadeh, S., OT

Sokolov, Eugene, 02

Soler Penadés, Jordi, OL

Stefancich, Marco, 17, 18

Terracciano, M., OV

Thomas, Fabrice, OB, OC

Ulliac, G., OC

Vannucci, A., 03

Vanwolleghem, Mathias, 15, 16

Vartapetov, Sergey K., IA

Vasinek, Vladimír, OK

Von den Driesch, N., OT

Wahlbrink, T., 07

Wang, Jian, 09

Wang, Junjia, OM

Wang, Shurui, Ol, OJ

Wangüemert-Pérez, Juan Gonzalo, OL

Witzens, J., OT

Wolf, S., 07

XU, Dan-Xia, OI, OJ, OK

Ye, Winnie N., OK

Yoshimura, Tetsuzo, 05

Zhang, Z., 03 


\section{Conference Committee}

Symposium Chairs

Jiri Homola, Institute of Photonics and Electronics of the ASCR, v.v.i. (Czech Republic)

Chris Edwards, Central Laser Facility, Science and Technology

Facilities Council (United Kingdom)

Mike Dunne, SLAC National Accelerator Laboratory (United States) and Linac Coherent Light Source (United States)

Ivo Rendina, Istituto per la Microelettronica e Microsistemi (Italy)

Honorary Symposium Chair

Miroslav Miller, Institute of Photonics and Electronics of the ASCR, v.v.i. (Czech Republic)

Conference Chairs

Pavel Cheben, National Research Council Canada (Canada) Jirí Čtyroký, Institute of Photonics and Electronics of the ASCR, v.v.i. (Czech Republic)

Iñigo Molina-Fernández, Universidad de Málaga (Spain)

\section{Conference Programme Committee}

Roel G. Baets, Universiteit Gent (Belgium)

Trevor Mark Benson, The University of Nottingham (United Kingdom)

Hung-Chun Chang, National Taiwan University (Taiwan)

Christopher R. Doerr, Acacia Communications Inc. (United States)

Romuald Houdré, Ecole Polytechnique Fédérale de Lausanne (Switzerland)

Raman Kashyap, Ecole Polytechnique de Montréal (Canada) Christophe Kazmierski, III-V Laboratoire (France)

Philippe Lalanne, Institut d'Optique Graduate School (France)

Xaveer J. M. Leijtens, Technische Universiteit Eindhoven (Netherlands)

Goran Z. Mashanovich, University of Southampton (United Kingdom)

Andrea I. Melloni, Politecnico di Milano (Italy)

Jarmila Müllerová, University of Žilina (Slovakia)

Martin Schell, Fraunhofer-Institut für Nachrichtentechnik HeinrichHertz-Institut (Germany)

Laurent Vivien, Institut d'Électronique Fondamentale (France)

Lech Wosinski, KTH Royal Institute of Technology (Sweden)

Dan-Xia Xu, National Research Council Canada (Canada) 
Session Chairs

1 Photonic Integration

Jirí Čtyroký, Institute of Photonics and Electronics of the ASCR, v.v.i. (Czech Republic)

2 Devices and Circuits I

Jiř́ Čtyroký, Institute of Photonics and Electronics of the ASCR, v.v.i. (Czech Republic)

3 Devices and Circuits II

David J. Moss, The University of Sydney (Australia)

4 Photonic Crystals and Plasmonic Devices

Andrea I. Melloni, Politecnico di Milano (Italy)

5 Subwavelength Structures

Iñigo Molina-Fernández, Universidad de Málaga (Spain)

6 Theory, Simulation, and Design

Min Qiu, KTH Royal Institute of Technology (Sweden)

7 Materials and Fabrication

Toshihiko Baba, Yokohama National University (Japan) 